\title{
Nonreciprocal Directional Dichroism and Toroidalmagnons in Helical Magnets
}

\author{
Shin Miyahara ${ }^{1}$ and Nobuo Furukawa ${ }^{1,2}$ \\ ${ }^{1}$ Multiferroics Project (MF), ERATO, Japan Science and Technology Agency (JST), \\ c/o Department of Applied Physics, The University of Tokyo, Bunkyo-ku, Tokyo 113-8656, Japan \\ ${ }^{2}$ Department of Physics and Mathematics, Aoyama Gakuin University, Sagamihara 229-8558, Japan
}

\begin{abstract}
We investigate a dynamical magnetoelectric effect due to a magnetic resonance in helical spin structures through the coupling between magnetization and electric polarization via a spin current mechanism. We show that the magnon has both the dynamical magnetic moment $\Delta M^{\omega}$ and the electric moment $\Delta P^{\omega}\left(\perp \Delta M^{\omega}\right)$, i.e., a dynamical toroidal moment, under external magnetic fields, and thus it is named the toroidalmagnon. The toroidalmagnon exists in most conical spin structures owing to the generality of the spin current mechanism. In the absorption of electromagnetic waves, the toroidalmagnon excitation process generally induces a nonreciprocal directional dichroism as a consequence of an interference of the magnetic and electric responses.
\end{abstract}

KEYWORDS: multiferroics, toroidalmagnon, nonreciprocal directional dichroism, spin current mechanism, frustration

Electromagnons are magnons that accompany an electric dipole moment. Electromagnon excitations are thus electro-active, i.e., they are induced by the electric component of light, and therefore are classified as dynamical magnetoelectric phenomena. ${ }^{1-3}$ Electromagnons are observed as resonances in optical spectroscopy at terahertz frequencies in various materials, e.g., $R \mathrm{MnO}_{3},{ }^{1,4,5} \quad R \mathrm{Mn}_{2} \mathrm{O}_{5},{ }^{6} \mathrm{Ba}_{2} \mathrm{Mg}_{2} \mathrm{Fe}_{12} \mathrm{O}_{22},{ }^{7}$ $\mathrm{CuFe}_{1-\mathrm{x}} \mathrm{Ga}_{\mathrm{x}} \mathrm{O}_{2},{ }^{8}$ and $\mathrm{Ba}_{2} \mathrm{CoGe}_{2} \mathrm{O}_{7} \cdot{ }^{9}, 10$ Their possible applications have been discussed.. ${ }^{3,11,12}$

Electro-active magnetic resonance (MR) processes can be understood by spin-dependent electric polarizations $\mathbf{P}\left(\left\{\mathbf{S}_{i}\right\}\right) .{ }^{13,14}$ Through the coupling between the electric component of light $\mathbf{E}^{\omega}$ and $\mathbf{P}\left(\left\{\mathbf{S}_{i}\right\}\right)$, a spin structure is modulated by $\mathbf{E}^{\omega}$. When such modulation induces a single-magnon excitation in an ordered magnet, the excitation is called the electromagnon. Thus far, the electromagnons induced through the spin current, ${ }^{2,15,16} \mathrm{ex}-$ change striction, $, 17,18$ and metal ligand hybridization mechanisms ${ }^{19}$ have been proposed.

Although most of these mechanisms have restrictions with respect to lattice symmetry, the spin current mechanism can generally induce electric polarization in cycloidal spin configurations, ${ }^{20,21}$ irrespective of local lattice symmetries. ${ }^{22}$ Let us consider a helical magnet with a cycloidal spin configuration $\mathbf{h} \perp \mathbf{q}_{0}$, where $\mathbf{q}_{0}$ is the propagation vector of the magnetic structure and $\mathbf{h}=\mathbf{S}_{i} \times \mathbf{S}_{j}$ gives the helicity vector. Here, $\mathbf{S}_{i}$ and $\mathbf{S}_{j}$ are spins on adjacent sites along the $\mathbf{q}_{0}$ direction. The spin current mechanism induces the electric polarization $\mathbf{P} \sim\left(\mathbf{h} \times \mathbf{q}_{0}\right)$. In ref. 2 , it is discussed that the uniform rotation of the helicity vector around the $q_{0}$ direction accompanies the dynamical fluctuation of the electric polarization; thus, such magnon excitation is electro-active. Since such magnetic excitation is the Nambu-Goldstone mode, the electromagnon should ubiquitously be identified in cycloidal spin systems.

Another novel feature in the presence of dynamical magnetoelectric effects is the nonreciprocal directional dichroism (NDD) where absorption intensity depends on the direction of an electromagnetic wave propagation vector. ${ }^{23,24}$ Recently, NDD in MR has been observed in $\mathrm{Ba}_{2} \mathrm{CoGe}_{2} \mathrm{O}_{7}{ }^{9}$ owing to the electro-active magnetic excitation process via the metal ligand hybridization mechanisms. ${ }^{19,25}$ Since the observation of NDD at a magnon resonance is a direct evidence of the presence of dynamical magnetoelectric effects including an electromagnon process, the investigation of NDD is important for understanding the magnetoelectric properties of the system. The metal ligand hybridization mechanism, however, depends on the local lattice structure; thus, NDD due to such a mechanism should be observed in a limited class of materials. On the other hand, in the generic electromagnons induced through the spin current mechanism in cycloidal spin structures, NDD is not observed with symmetrical restriction, despite the fact that dynamical magnetoelectric couplings exist. ${ }^{2}$

In this Letter, we discuss that NDD should ubiquitously be observed if one applies a static magnetic field to helical magnets with spin current couplings. We clarify the existence of magnetic excitations that accompany both magnetic and electric moments, which contribute to dynamical magnetoelectric effects at their resonances. We discuss the existence of the dynamical toroidal moment in magnon excitation as the origin of NDD.

Let us first review NDD briefly. ${ }^{9,19}$ In the presence of dynamical magneto-electric effects, we have

$$
\begin{aligned}
\mathbf{B}^{\omega} & =\left(\hat{\mu}^{\infty}+\hat{\chi}^{\mathrm{mm}}\right) \mu_{0} \mathbf{H}^{\omega}+\hat{\chi}^{\mathrm{me}} \sqrt{\epsilon_{0} \mu_{0}} \mathbf{E}^{\omega}, \\
\mathbf{D}^{\omega} & =\left(\hat{\epsilon}^{\infty}+\hat{\chi}^{\mathrm{ee}}\right) \epsilon_{0} \mathbf{E}^{\omega}+\hat{\chi}^{\mathrm{em}} \sqrt{\epsilon_{0} \mu_{0}} \mathbf{H}^{\omega},
\end{aligned}
$$

where $\hat{\chi}^{\mathrm{mm}}$ and $\hat{\chi}^{\mathrm{ee}}$ are dynamical magnetic and electric susceptibility tensors, respectively, and $\hat{\chi}^{\mathrm{me}}$ and $\hat{\chi}^{\mathrm{em}}$ are dynamical magnetoelectric susceptibility tensors. As an example, let us consider a linearly polarized light with $\mathbf{E}^{\omega}\left\|x, \mathbf{H}^{\omega}\right\| y$, and the propagation vector $\mathbf{k} \| z$. In the presence of off-diagonal magnetoelectric susceptibilities, 
the interferences between $E_{x}^{\omega}$ and $H_{y}^{\omega}$ in $\mathbf{B}^{\omega}$ and $\mathbf{D}^{\omega}$ given by eqs. (1) and (2) are affected by the relative sign of $E_{x}^{\omega}$ and $H_{y}^{\omega}$ and thus by the direction of $\mathbf{k}$ since $\omega \mu_{0} \mathbf{H}^{\omega}=\mathbf{k} \times \mathbf{E}^{\omega}$. For simplicity, we assume isotropic and constant $\epsilon^{\infty}$ and $\mu^{\infty}$. From the Maxwell equation, the complex refractive index $N$ is approximated as

$$
\begin{aligned}
N\left(s_{k}\right) \sim & \sqrt{\left[\epsilon_{\infty}+\chi_{x x}^{\mathrm{ee}}(\omega)\right]\left[\mu_{\infty}+\chi_{y y}^{\mathrm{mm}}(\omega)\right]} \\
& +s_{k}\left[\chi_{y x}^{\mathrm{me}}(\omega)+\chi_{x y}^{\mathrm{em}}(\omega)\right] / 2
\end{aligned}
$$

as a function of the direction of the propagation vector $s_{k}=k_{z} /\left|k_{z}\right|$. The absorption coefficient is proportional to $\operatorname{Im} N\left(s_{k}\right)$. Therefore, the absorption intensity depends on the light propagation direction $s_{k}$ and their difference is proportional to $\operatorname{Im} N(+)-\operatorname{Im} N(-)=\operatorname{Im}\left[\chi_{y x}^{\operatorname{me}}(\omega)+\right.$ $\left.\chi_{x y}^{\mathrm{em}}(\omega)\right]$. In general, when we have off-diagonal magnetoelectric susceptibilities,

$$
\operatorname{Im}\left[\chi_{\beta \alpha}^{\mathrm{me}}(\omega)+\chi_{\alpha \beta}^{\mathrm{em}}(\omega)\right] \neq 0
$$

the system exhibits NDD for an incident light with $E_{\alpha}^{\omega}$ and $H_{\beta}^{\omega}$ for $\alpha, \beta=x, y, z(\alpha \perp \beta)$.

Using the Kubo formula, the dynamical susceptibilities $\chi_{\gamma \tau}^{\mathrm{mm}}(\omega), \chi_{\gamma \tau}^{\mathrm{ee}}(\omega), \chi_{\gamma \tau}^{\mathrm{me}}(\omega)$, and $\chi_{\gamma \tau}^{\mathrm{em}}(\omega)$ for $\gamma, \tau=x, y, z$ are described as

$$
\begin{aligned}
\chi_{\gamma \tau}^{\mathrm{mm}}(\omega) & =\frac{\mu_{0}}{\hbar N V} \sum_{n} \frac{\left\langle 0\left|\Delta M_{\gamma}\right| n\right\rangle\left\langle n\left|\Delta M_{\tau}\right| 0\right\rangle}{\omega_{n 0}-\omega-i \delta} \\
\chi_{\gamma \tau}^{\mathrm{ee}}(\omega) & =\frac{1}{\hbar N V \epsilon_{0}} \sum_{n} \frac{\left\langle 0\left|\Delta P_{\gamma}\right| n\right\rangle\left\langle n\left|\Delta P_{\tau}\right| 0\right\rangle}{\omega_{n 0}-\omega-i \delta} \\
\chi_{\gamma \tau}^{\mathrm{me}}(\omega) & =\frac{1}{\hbar N V} \sqrt{\frac{\mu_{0}}{\epsilon_{0}}} \sum_{n} \frac{\left\langle 0\left|\Delta M_{\gamma}\right| n\right\rangle\left\langle n\left|\Delta P_{\tau}\right| 0\right\rangle}{\omega_{n 0}-\omega-i \delta},( \\
\chi_{\gamma \tau}^{\mathrm{em}}(\omega) & =\frac{1}{\hbar N V} \sqrt{\frac{\mu_{0}}{\epsilon_{0}}} \sum_{n} \frac{\left\langle 0\left|\Delta P_{\gamma}\right| n\right\rangle\left\langle n\left|\Delta M_{\tau}\right| 0\right\rangle}{\omega_{n 0}-\omega-i \delta} .
\end{aligned}
$$

Here, $|0\rangle$ is a ground state with the eigenenergy $E_{0},|n\rangle$ is an $n$-th magnetic excitation state with $E_{n}$, and $\hbar \omega_{n 0}=$ $E_{n}-E_{0} . V$ is the unit volume per spin and $N$ is the number of spins. Magnetization is defined by

$$
\mathbf{M}=\sum_{i} g \mu_{B} \mathbf{S}_{i}
$$

and $\Delta M_{\gamma}$ is the $\gamma$-component of the magnetization fluctuations from the ground state. We only consider the contributions to the electric polarization induced by the spin current mechanism, ${ }^{20}$

$$
\mathbf{P}=\lambda \sum_{i j} \mathbf{e}_{i j} \times\left(\mathbf{S}_{i} \times \mathbf{S}_{j}\right),
$$

which exists in generic systems in the presence of spinorbit couplings. Here, $\mathbf{e}_{i j} \| y$ is the unit vector connecting the nearest-neighbor (n.n.) spins $\mathbf{S}_{i}$ and $\mathbf{S}_{j}$, and $\lambda$ is a coupling constant that is proportional to spin-orbit couplings. $\Delta P_{\gamma}$ in eqs. (6)-(8) is the fluctuation of $\mathbf{P}$.

From eqs. (5) and (6), we see that electromagnon excitation exists if $\left\langle n\left|\Delta P_{\gamma}\right| 0\right\rangle \neq 0$ for a one-magnon excitation state $|n\rangle$, while a conventional MR occurs for $\left\langle n\left|\Delta M_{\gamma}\right| 0\right\rangle \neq 0$. Furthermore, from eqs. (4), (7), and (8), we have NDD at an electromagnon resonance if $\operatorname{Re}\left\langle 0\left|\Delta M_{\tau}\right| n\right\rangle\left\langle n\left|\Delta P_{\gamma}\right| 0\right\rangle \neq 0$ with $\tau \perp \gamma$ being satisfied
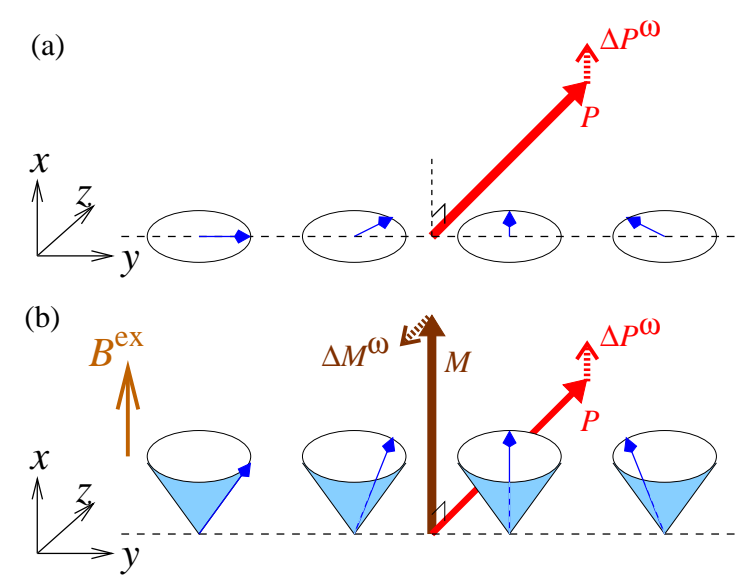

Fig. 1. (Color online) (a) Schematic view of a $y z$-cycloidal state with $\mathbf{P} \| z$ induced by the spin current mechanism. The spin rotation around the $y$-axis accompanies $\Delta \mathbf{P}^{\omega} \| x$. (b) $y z$-cycloidal conical state with $\mathbf{P} \| z$ and $\mathbf{M} \| x$. The tilting of the cones around the $y$-axis creates $\Delta \mathbf{P}^{\omega} \| x$ and $\Delta \mathbf{M}^{\omega} \| z$.

for an excited state $|n\rangle$.

We consider a $y z$-cycloidal screw magnet, i.e., $\mathbf{q}_{0} \| y$ and $\mathbf{h} \| x$, at $\mathbf{B}^{\mathrm{ex}}=0$, as an example. This structure accompanies the electric polarization $\mathbf{P} \| z$. Through the Nambu-Goldstone mode, which modulates the spin structure in such a way that the cycloidal plane rotates along the $y$-axis, the modulation of the spontaneous electric polarization $\Delta \mathbf{P}^{\omega} \| x$ occurs, as shown in Fig. 1(a). Then, such a mode is activated by $\mathbf{E}^{\omega} \| x .^{2}$ Since the modulation of the spin structure does not induce a uniform magnetization, such an electromagnon mode should not be magneto-active, i.e., $\Delta \mathbf{M}=0$. Thus, the electromagnon resonance should not accompany NDD.

On the other hand, under the external magnetic field $\mathbf{B}^{\mathrm{ex}} \| x$, spins give a conical structure, as depicted in Fig. 1(b). Here, we have both the electric polarization $\mathbf{P} \| z$ and the magnetization $\mathbf{M} \| x$ in the ground state, and thus have a static toroidal moment defined by $\mathbf{T}=\mathbf{P} \times \mathbf{M} .^{26}$ If we consider an excited state $|n\rangle$ where the conical structure is dynamically tilted along the $y$-axis, ${ }^{15}$ both $\Delta \mathbf{P}^{\omega} \| x$ and $\Delta \mathbf{M}^{\omega} \| z$ exist coherently. When the system is excited by an incident light with $\mathbf{E}^{\omega} \| x$ and $\mathbf{H}^{\omega} \| z$, the tilting motion of the conical structure of the spins around the $y$-axis exhibits $\operatorname{Re}\left\langle 0\left|\Delta M_{z}\right| n\right\rangle\left\langle n\left|\Delta P_{x}\right| 0\right\rangle \neq 0$ and thus NDD. In a semiclassical picture, NDD is observed at a resonance owing to a magnon with the dynamical toroidal moment $\mathbf{T}^{d}=\Delta \mathbf{P}^{\omega} \times \Delta \mathbf{M}^{\omega}$, i.e., a toroidalmagnon, irrespective of lattice dimensions or symmetries. Note that the NDD effect is linearly proportional to $\Delta P$. Thus, in the weak coupling region, it may be detected more easily than the electromagnon absorption itself, which is proportional to $(\Delta P)^{2}$.

To obtain microscopic details, we investigate a frustrated (quasi-)one-dimensional Heisenberg model,

$$
\mathcal{H}=J_{1} \sum_{\text {n.n. }} \mathbf{S}_{i} \cdot \mathbf{S}_{j}+J_{2} \sum_{\text {n.n.n. }} \mathbf{S}_{i} \cdot \mathbf{S}_{j},
$$

as a canonical spin model for helimagnets. Here, $\mathbf{S}_{i}$ is 
a spin operator on the $i$-th site. We assume the ferromagnetic n.n. spin exchange interaction $J_{1}$ and antiferromagnetic next-nearest-neighbor (n.n.n.) interaction $J_{2}$, i.e., $J_{1}<0$ and $J_{2}>0$. In the absence of the external magnetic field $B^{\mathrm{ex}}$, the helical spin state is the ground state in the parameter range $J_{2} /\left|J_{1}\right|>0.25$ for classical spins owing to the frustration. Hereafter, we consider $\mathbf{q}_{0} \| y$ as the propagation vector, i.e., the helical spin structure along the $y$-direction has a $2 \pi / q_{0}$ period, where $\cos q_{0}=-J_{1} / 4 J_{2}$. When $\mathbf{B}^{\mathrm{ex}}$ is applied, a conical spin state emerges. The periodicity of the helical structure does not depend on $\mathbf{B}^{\mathrm{ex}} \cdot{ }^{27}$ We apply the lowest order spinwave expansion from the ground state.

First, we consider a helical magnet with $\mathbf{B}^{\mathrm{ex}} \perp \mathbf{q}_{0}$. For $\mathbf{B}^{\mathrm{ex}} \| x$, we have $y z$-cycloidal and conical spin structures with the helicity vector $\mathbf{h}=\mathbf{S}_{i} \times \mathbf{S}_{i+1} \| x$. The ground state spin structure is described as

$$
\mathbf{S}_{i}=\left(S \cos \theta, S \sin \theta \sin \left(q_{0} r_{i}\right), S \sin \theta \cos \left(q_{0} r_{i}\right)\right),
$$

where the cone angle $\theta$ is defined as

$$
2 S \cos \theta=\frac{g \mu_{B} B_{x}^{\mathrm{ex}}}{J_{1}\left(1-\cos q_{0}\right)+J_{2}\left(1-\cos 2 q_{0}\right)} .
$$

Note that $\theta \rightarrow \pi / 2$ for $B_{x}^{\mathrm{ex}} \rightarrow 0$ such that we recover a pure helical structure. We have $\mathbf{P} \| z$ and $\mathbf{M} \| x$ in the ground state.

Following the procedure indicated in ref. 27, we use the linear spin wave theory in eq. (11). We define the local coordinates where the $\xi$-direction is aligned to the equilibrium spin directions:

$S_{i}^{x}=-S_{i}^{\zeta} \sin \theta+S_{i}^{\xi} \cos \theta$

$S_{i}^{y}=\left(S_{i}^{\zeta} \cos \theta+S_{i}^{\xi} \sin \theta\right) \sin \left(q_{0} r_{i}\right)+S_{i}^{\eta} \cos \left(q_{0} r_{i}\right)$,

$S_{i}^{z}=\left(S_{i}^{\zeta} \cos \theta+S_{i}^{\xi} \sin \theta\right) \cos \left(q_{0} r_{i}\right)-S_{i}^{\eta} \sin \left(q_{0} r_{i}\right)$.

Hereafter, we choose $q_{0}>0$ for the ground state. Then, the Hamiltonian (11) can be written in terms of local $\zeta$, $\eta$, and $\xi$ coordinates by using $S_{i}^{+}, S_{i}^{-}$, and $S_{i}^{\xi}$. For spin operators, we consider the Holstein-Primakoff approximations:

$$
S_{i}^{+}=\sqrt{2 S} a_{i}, \quad S_{i}^{-}=\sqrt{2 S} a_{i}^{\dagger}, \quad S_{i}^{\xi}=S-a_{i}^{\dagger} a_{i} .
$$

By the Fourier transformations of $a_{i}$ and $a_{i}^{\dagger}$, the linear spinwave Hamiltonian in the $q$ space is written as

$$
\mathcal{H}=E_{0}+S \sum_{q}\left[2 A_{q} a_{q}^{\dagger} a_{q}+B_{q}\left(a_{q}^{\dagger} a_{-q}^{\dagger}+a_{q} a_{-q}\right)\right],
$$

where

$$
\begin{aligned}
A_{q}= & -J_{1}\left\{\cos q_{0}+\cos \theta \sin q_{0} \sin q\right. \\
& \left.-\frac{1}{2}\left[\left(1+\cos ^{2} \theta\right) \cos q_{0}+\sin ^{2} \theta\right] \cos q\right\} \\
& -J_{2}\left\{\cos 2 q_{0}+\cos \theta \sin 2 q_{0} \sin 2 q\right. \\
& \left.-\frac{1}{2}\left[\left(1+\cos ^{2} \theta\right) \cos 2 q_{0}+\sin ^{2} \theta\right] \cos 2 q\right\}, \\
B_{q}= & \frac{J_{1}}{2} \sin ^{2} \theta\left(1-\cos q_{0}\right) \cos q \\
& +\frac{J_{2}}{2} \sin ^{2} \theta\left(1-\cos 2 q_{0}\right) \cos 2 q .
\end{aligned}
$$

Table I. Non-zero coefficients $f_{\tau}^{\alpha}(q)$ for $\tau=x, y, z$ and $\alpha=\mathrm{m}$,e in a $y z$-cycloidal conical spin structure with qo $\| y$ and $\mathbf{B}^{\mathrm{ex}}\|\mathbf{h}\| x$.

$$
\begin{array}{ll}
\Delta M_{y} & f_{y}^{\mathrm{m}}\left( \pm q_{0}\right)=i\left[(1 \pm \cos \theta) c_{q_{0}}+(1 \mp \cos \theta) s_{q_{0}}\right] / 4 \\
\Delta M_{z} & f_{z}^{\mathrm{m}}\left( \pm q_{0}\right)= \pm\left[(1 \pm \cos \theta) c_{q_{0}}+(1 \mp \cos \theta) s_{q_{0}}\right] / 4 \\
\hline \Delta P_{x} & f_{x}^{\mathrm{e}}\left( \pm q_{0}\right)=-\left(c_{q_{0}}-s_{q_{0}}\right) \sin ^{2} \theta \sin q_{0} / 2
\end{array}
$$

Here, the magnon wave vector $q$ is defined in an extended Brillouin zone to clarify distinctions for the magnon modes. The Hamiltonian (18) can be diagonalized by the standard Bogoliubov transformation,

$$
\alpha_{q}^{\dagger} \equiv c_{q} a_{q}^{\dagger}+s_{q} a_{-q}, \quad \alpha_{q} \equiv s_{q} a_{-q}^{\dagger}+c_{q} a_{q}
$$

where the coefficients $c_{q}\left(c_{q}=c_{-q}\right)$ and $s_{q}\left(s_{q}=s_{-q}\right)$ are given by

$$
c_{q}=\frac{2 B_{q}}{\sqrt{4 B_{q}^{2}-C_{q}^{2}}}, \quad s_{q}=\frac{C_{q}}{\sqrt{4 B_{q}^{2}-C_{q}^{2}}},
$$

for $C_{q}=A_{q}+A_{-q}-\left[\left(A_{q}+A_{-q}\right)^{2}-4 B_{q}^{2}\right]^{1 / 2}$. We obtain

$$
\mathcal{H}=\sum_{q} \hbar \omega_{q} \alpha_{q}^{\dagger} \alpha_{q}+\text { const }
$$

where the spin wave frequencies are

$$
\hbar \omega_{q}=S\left[\left(A_{q}-A_{-q}\right)+\sqrt{\left(A_{q}+A_{-q}\right)^{2}-4 B_{q}^{2}}\right] .
$$

For the helical spin state, i.e., $B^{\mathrm{ex}}=0, A_{q}=A_{-q}$ so that the magnons at $q$ and $-q$ are degenerate. On the other hand, the external magnetic field lifts the degeneracy owing to $A_{q} \neq A_{-q}$, i.e., $\hbar \omega_{q} \neq \hbar \omega_{-q}$ for $B^{\mathrm{ex}} \neq 0$.

When the spinwave expansion of $\mathbf{P}$ contains linear terms of magnon creation and annihilation operators $\alpha_{q}^{\dagger}$ and $\alpha_{q}$, the electric component of light creates a oneelectromagnon excited state, through the perturbation term $\mathcal{H}^{\prime}=-\mathrm{E}^{\omega} \Delta P$. In the present model, we apply eqs. (17) and (20) to $\mathbf{P}$ and $\mathbf{M}$. Both the magnetization (9) and the electric polarization (10) can be described as

$$
\begin{aligned}
\Delta M_{\tau} & \sim \sum_{q} g \mu_{B} \sqrt{2 S N}\left[f_{\tau}^{\mathrm{m}}(q) \alpha_{q}^{\dagger}+f_{\tau}^{\mathrm{m} *}(q) \alpha_{q}\right] \\
\Delta P_{\tau} & \sim \sum_{q} \lambda S \sqrt{2 S N}\left[f_{\tau}^{\mathrm{e}}(q) \alpha_{q}^{\dagger}+f_{\tau}^{\mathrm{e} *}(q) \alpha_{q}\right] .
\end{aligned}
$$

Non-zero coefficients for the the lowest order spinwave expansion $f_{\tau}^{\alpha}(q)$ for $\alpha=\mathrm{e}, \mathrm{m}$ and $\tau=x, y, z$ are summarized in Table I. We only have non-zero contributions from $q= \pm q_{0}$ magnons, which correspond to the electromagnons discussed in ref. 2. Note that, within the magnetic Brillouin zone, $q= \pm q_{0}$ locates at the $\Gamma$ point. At $B^{\text {ex }}=0$, these $q= \pm q_{0}$ Nambu-Goldstone modes are gapless and thus do not show resonance in reality. However, under $B^{\mathrm{ex}} \neq 0$, the $q= \pm q_{0}$ magnon excitation has the gap $\hbar \omega_{ \pm q_{0}}$ due to Zeeman energy. Thus, the magnetoelectric resonance of the magnon can be observed in a finite frequency range where a conventional MR is observed.

Within a linear spinwave treatment, the susceptibili- 
ties $\chi_{\gamma \tau}^{\mathrm{mm}}(\omega), \chi_{\gamma \tau}^{\mathrm{ee}}(\omega), \chi_{\gamma \tau}^{\mathrm{me}}(\omega)$, and $\chi_{\gamma \tau}^{\mathrm{em}}(\omega)$ are given as

$$
\chi_{\gamma \tau}^{\alpha \beta}(\omega)=\frac{C^{\alpha \beta}}{\hbar V} \sum_{q} \frac{f_{\gamma}^{\alpha *}(q) f_{\tau}^{\beta}(q)}{\omega_{q}-\omega-i \delta} \quad\left(\begin{array}{c}
\alpha, \beta=\mathrm{e}, \mathrm{m} \\
\gamma, \tau=x, y, z
\end{array}\right) \text {, }
$$

where $C^{\mathrm{mm}}=2 S\left(g \mu_{B}\right)^{2} \mu_{0}, C^{\mathrm{ee}}=2 S^{3} \lambda^{2} / \epsilon_{0}$, and $C^{\mathrm{me}}=$ $C^{\mathrm{em}}=2 S^{2} \lambda g \mu_{B} \sqrt{\mu_{0} / \epsilon_{0}}$. Namely, when $\left|f_{\tau}^{\mathrm{m}}(q)\right|^{2} \neq 0$, the resonance due to the magnon excitation at $\omega=\omega_{q}$ is induced by $H_{\tau}^{\omega}$, while $\left|f_{\gamma}^{\mathrm{e}}(q)\right|^{2} \neq 0$ gives an electromagnon excited by $E_{\gamma}^{\omega}$. For a non degenerate magnon $\left(\omega_{q} \neq \omega_{-q}\right), \operatorname{Re}\left[f_{\tau}^{\mathrm{m} *}(q) f_{\gamma}^{\mathrm{e}}(q)\right] \neq 0(\tau \perp \gamma)$ ensures NDD for electromagnetic waves with $H_{\tau}^{\omega}$ and $E_{\gamma}^{\omega}$ with an intensity $\propto C^{\mathrm{me}}$.

Since $\left|f_{x}^{\mathrm{e}}\left( \pm q_{0}\right)\right|^{2} \neq 0$ as shown in Table I, these magnons give electric resonances for $\chi_{x x}^{\mathrm{ee}}(\omega)$ at $\omega=\omega_{q_{0}}$ and $\omega_{-q_{0}}$. This indeed indicates that MRs can be driven by $\mathbf{E}^{\omega} \| x$ as absorptions by electromagnons. We also see $\left|f_{y}^{\mathrm{m}}\left( \pm q_{0}\right)\right|^{2}=\left|f_{z}^{\mathrm{m}}\left( \pm q_{0}\right)\right|^{2} \neq 0$; thus, $\mathbf{H}^{\omega} \perp x$ induces MR at the $\mathbf{q}= \pm q_{0}$ magnon energy. ${ }^{27}$ These modes show NDD for an incident light with $\mathbf{E}^{\omega}\left\|x, \mathbf{H}^{\omega}\right\| z$, and $\mathbf{k} \| y$, since $\chi_{z x}^{\mathrm{me}}\left(\omega_{ \pm q_{0}}\right)=\chi_{x z}^{\mathrm{em}}\left(\omega_{ \pm q_{0}}\right) \propto f_{z}^{\mathrm{m} *}\left( \pm q_{0}\right) f_{x}^{\mathrm{e}}\left( \pm q_{0}\right)+$ $f_{x}^{\mathrm{e} *}\left( \pm q_{0}\right) f_{z}^{\mathrm{m}}\left( \pm q_{0}\right) \neq 0$. In this manner, the electromagnon at $q= \pm q_{0}$ has a dynamical toroidal moment $\mathbf{T}^{d} \neq 0$ and thus is identified as a toroidalmagnon. At $B^{\text {ex }}=0$, however, the $\pm q_{0}$ magnons become degenerate; thus, the cross-correlated effects at the $\omega=\omega_{q_{0}}$ and $\omega_{-q_{0}}$ peaks merge to cancel themselves: $\chi_{z x}^{\text {me }}\left(\omega_{q_{0}}\right) \propto$ $f_{z}^{\mathrm{m} *}\left(q_{0}\right) f_{x}^{\mathrm{e}}\left(q_{0}\right)+f_{z}^{\mathrm{m} *}\left(-q_{0}\right) f_{x}^{\mathrm{e}}\left(-q_{0}\right)=0$.

Other components of susceptibilities are also obtained from Table I. Magnons at $q= \pm q_{0}$ contribute to the offdiagonal magnetic susceptibility $\chi_{y z}^{\mathrm{mm}}(\omega)=-\chi_{z y}^{\mathrm{mm}}(\omega)$ at $B^{\text {ex }} \neq 0$, which give a conventional Faraday rotation. For magnetoelectric susceptibilities, $\chi_{y x}^{\mathrm{me}}(\omega)$ and $\chi_{x y}^{\mathrm{em}}(\omega)$ are also non-zero. However, they do not cause NDD since $\operatorname{Im}\left[\chi_{y x}^{\mathrm{me}}(\omega)+\chi_{x y}^{\mathrm{em}}(\omega)\right]=0 .{ }^{28}$

By reversing the direction of the external magnetic fields $B^{\text {ex }} \rightarrow-B^{\text {ex }}, \chi_{z x}^{\text {me }}\left(\omega_{ \pm q_{0}}\right) \rightarrow-\chi_{z x}^{\text {me }}\left(\omega_{\mp q_{0}}\right)$ is obtained owing to $\theta \rightarrow \pi-\theta$ and $\hbar \omega_{ \pm q_{0}} \rightarrow \hbar \omega_{\mp q_{0}}$. Thus, reversing the light propagation direction is equivalent to reversing the external magnetic field direction for the absorption process.

Next, we investigate the case $\mathbf{B}^{\mathrm{ex}}\left\|\mathbf{q}_{0}\right\| y$, where a proper conical spin state is stabilized, as shown in Fig. 2. Note that the ground state does not have a spontaneous electric polarization owing to $\mathbf{e}_{i j}\|\mathbf{h}\| y$ such that $\mathbf{T}=\mathbf{P} \times \mathbf{M}=0$ statically. If we consider an excitation state where helical structures are uniformly tilted along the $z$-axis, i.e., $\mathbf{h}$ is rotated around the $z$-axis to create $\Delta \mathbf{h} \| x$, the electric polarization $\Delta \mathbf{P}^{\omega} \sim\left(\mathbf{e}_{i j} \times \Delta \mathbf{h}\right) \| z$ is induced, which couples to the external electric field $\mathbf{E}^{\omega} \| z$. Furthermore, in the case of $\mathbf{B}^{\text {ex }} \neq 0, \Delta \mathbf{M}^{\omega} \| x$ is also seen in this excited state. Thus, the mode is accompanied by the dynamical toroidal moment $\mathbf{T}^{d} \neq 0$ even in the absence of the static $\mathbf{T}$, such that NDD for an incident light with $\mathbf{E}^{\omega} \| z$ and $\mathbf{H}^{\omega} \| x$ is expected. The tilting of the conical structure along the $x$-axis is symmetry-wise equivalent. This excitation state will induce $\Delta \mathbf{P}^{\omega} \| x$ and $\Delta \mathbf{M}^{\omega} \| z$, and NDD for an incident light with $\mathbf{E}^{\omega} \| x$ and $\mathbf{H}^{\omega} \| z$ is similarly expected.

For details, we perform a similar calculation for the

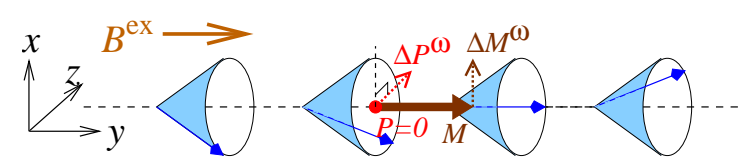

Fig. 2. (Color online) Schematic view of a $x z$-proper conical state with $\mathbf{M} \| y$ but $\mathbf{P}=0$. The tilting of the cones around the $z$-axis creates $\Delta \mathbf{P}^{\omega} \| z$ and $\Delta \mathbf{M}^{\omega} \| x$.

Table II. Non-zero coefficients $f_{\tau}^{\alpha}(q)$ for $\tau=x, y, z$ and $\alpha=\mathrm{m}, \mathrm{e}$ in an $x z$-proper conical spin structure with $\mathbf{q o}_{0}\left\|\mathbf{B}^{\mathrm{ex}}\right\| \mathbf{h} \| y$.

$$
\begin{array}{ll}
\Delta M_{x} & f_{x}^{\mathrm{m}}\left( \pm q_{0}\right)=-i\left[(1 \pm \cos \theta) c_{q_{0}}+(1 \mp \cos \theta) s_{q_{0}}\right] / 4 \\
\Delta M_{z} & f_{z}^{\mathrm{m}}\left( \pm q_{0}\right)= \pm\left[(1 \pm \cos \theta) c_{q_{0}}+(1 \mp \cos \theta) s_{q_{0}}\right] / 4 \\
\hline \Delta P_{x} & f_{x}^{\mathrm{e}}\left( \pm q_{0}\right)=-\left(c_{q_{0}}-s_{q_{0}}\right) \sin ^{2} \theta \sin q_{0} / 2 \\
\Delta P_{z} & f_{z}^{\mathrm{e}}\left( \pm q_{0}\right)=\mp i\left(c_{q_{0}}-s_{q_{0}}\right) \sin ^{2} \theta \sin q_{0} / 2
\end{array}
$$

spinwave expansions in a proper conical spin state with $\mathbf{q}_{\mathbf{0}}\left\|\mathbf{B}^{\mathrm{ex}}\right\| \mathbf{h} \| y$. We obtain $\Delta \mathbf{P}$ and $\Delta \mathbf{M}$ within the linear combinations of spinwave operators, as listed in Table II. We see that magnons with $q= \pm q_{0}$ are electro-active, owing to $\left|f_{x}^{\mathrm{e}}\left( \pm q_{0}\right)\right|^{2}=\left|f_{z}^{\mathrm{e}}\left( \pm q_{0}\right)\right|^{2} \neq 0$. Namely, these modes are electromagnons that couple to $\mathbf{E}^{\omega} \perp y$, irrespective of $\mathbf{B}^{\mathrm{ex}}$. Moreover, they are also magneto-active, since $\left|f_{x}^{\mathrm{m}}\left( \pm q_{0}\right)\right|^{2}=\left|f_{z}^{\mathrm{m}}\left( \pm q_{0}\right)\right|^{2} \neq 0$ at $\mathbf{B}^{\mathrm{ex}} \neq 0$.

Let us study NDD in this configuration in detail. For $\mathbf{E}^{\omega} \| z$ and $\mathbf{H}^{\omega} \| x$, we have $\operatorname{Im}\left[\chi_{x z}^{\mathrm{me}}\left(\omega_{ \pm q_{0}}\right)+\chi_{z x}^{\mathrm{em}}\left(\omega_{ \pm q_{0}}\right)\right] \neq$ 0 , owing to $f_{x}^{\mathrm{m} *}\left( \pm q_{0}\right) f_{z}^{\mathrm{e}}\left( \pm q_{0}\right)=f_{z}^{\mathrm{e} *}\left( \pm q_{0}\right) f_{x}^{\mathrm{m}}\left( \pm q_{0}\right) \neq 0$. Similarly, we have $\operatorname{Im}\left[\chi_{z x}^{\mathrm{me}}\left(\omega_{ \pm q_{0}}\right)+\chi_{x z}^{\mathrm{em}}\left(\omega_{ \pm q_{0}}\right)\right] \neq 0$ for $\mathbf{E}^{\omega} \| x$ and $\mathbf{H}^{\omega} \| z$. At $B^{\text {ex }}=0$, however, the crosscorrelated effects caused by $\pm q_{0}$-magnons are canceled owing to their degeneracy. Namely, for an incident light with $\mathbf{k} \| y$, we see NDD for any polarizations of $\mathbf{E}^{\omega}$ and $\mathbf{H}^{\omega}$, in the presence of $B^{\mathrm{ex}} \neq 0$. Note that this is the Faraday configuration $\mathbf{k} \| \mathbf{M}$ such that circularly polarized modes are the eigenmodes of the Maxwell equation in the presence of rotational symmetry along $\mathbf{k} \| \mathbf{M}$; thus, the so-called magnetochiral dichroism is observed. Details will be reported elsewhere. As in the cycloidal cone case, reversing the light propagation direction is equivalent to reversing the external magnetic field direction for the absorption process: $B^{\mathrm{ex}} \rightarrow-B^{\mathrm{ex}}(\theta \rightarrow \pi-\theta$ and $\left.\hbar \omega_{ \pm q_{0}} \rightarrow \hbar \omega_{\mp q_{0}}\right)$ induces $\chi_{z x}^{\mathrm{me}}\left(\omega_{ \pm q_{0}}\right) \rightarrow-\chi_{z x}^{\mathrm{me}}\left(\omega_{\mp q_{0}}\right)$ and $\chi_{x z}^{\mathrm{me}}\left(\omega_{ \pm q_{0}}\right) \rightarrow-\chi_{x z}^{\mathrm{me}}\left(\omega_{\mp q_{0}}\right)$.

Microscopically, NDD arises owing to the off-diagonal matrix elements of both the magnetic and electric dipole operators $\operatorname{Re}\left\langle 0\left|\Delta M_{\gamma}\right| n\right\rangle\left\langle n\left|\Delta P_{\tau}\right| 0\right\rangle(\gamma \neq \tau)$ [see eqs. (4), $(7)$, and (8)]. In general, the magnetic dipole operator is even (odd) under space-inversion $I$ (time-reversal $R$ ) symmetry operation, whereas the electric dipole operator is odd (even) under $I(R)$. Thus, in magnetic structures with $I$ and $R$ symmetry invariances, the real parts of the off-diagonal matrix elements are zero, i.e, NDD does not arise. In fact, the matrix elements in pure helical structures vanish owing to the $R$ symmetry invariance, and NDD is induced only in conical structures where $I$ and $R$ symmetries are broken.

To summarize, we investigated NDD that arises in conical spin structures via the spin-current mecha- 
nism. NDD is generally induced by the excitation of a magnon accompanied by a dynamical toroidal moment, i.e, toroidalmagnon. In a conical structure, the fluctuation of the dynamical toroidal moment is observed with cone oscillations, as shown in Figs. 1 and 2. Such modes are adiabatically connected to the $q= \pm q_{0}$ NambuGoldstone modes, and thus we conclude that a toroidalmagnon should ubiquitously exist in conical spin structures. Let us finally note that the observation of NDD via the magnon excitation in a perovskite helimagnet has been reported in ref. 29 quite recently, which is qualitatively explained by our theory.

\section{Acknowledgment}

We thank I. Kézsmárki, S. Bordács, Y. Takahashi, N. Kida, T. Arima, R. Shimano, N. Nagaosa, and Y. Tokura for fruitful discussion. This work is supported in part by Grants-In-Aid for Scientific Research from the Ministry of Education, Culture, Sports, Science and Technology (MEXT), Japan.

1) A. Pimenov, A. A. Mukhin, V. Y. Ivanov, V. D. Travkin, A. M. Balbashov, and A. Loidl: Nat. Phys. 2 (2006) 97.

2) H. Katsura, A. V. Balatsky, and N. Nagaosa: Phys. Rev. Lett. 98 (2007) 027203.

3) Y. Tokura and N. Kida: Phil. Trans. R. Soc. A 369 (2011) 3679.

4) N. Kida, Y. Takahashi, J. Lee, R. Shimano, Y. Yamasaki, Y. Kaneko, S. Miyahara, N. Furukawa, T. Arima, and Y. Tokura: J. Opt. Soc. Am. B 26 (2009) A35.

5) R. V. Aguilar, M. Mostovoy, A. B. Sushkov, C. L. Zhang, Y. J. Choi, S.-W. Cheong, and H. Drew: Phys. Rev. Lett. 102 (2009) 047203.

6) A. Sushkov, M. Mostovoy, R. Aguilar, S.-W. Cheong, and H. Drew: J. Phys.: Condens. Matter 20 (2008) 434210.

7) N. Kida, D. Okuyama, S. Ishiwata, Y. Taguchi, R. Shimano, K. Iwasa, T. Arima, and Y. Tokura: Phys. Rev. B 80 (2009) 220406.
8) S. Seki, N. Kida, S. Kumakura, R. Shimano, and Y. Tokura: Phys. Rev. Lett. 105 (2010) 097207.

9) I. Kézsmárki, N. Kida, H. Murakawa, S. Bordács, Y. Onose, and Y. Tokura: Phys. Rev. Lett. 106 (2011) 057403.

10) S. Bordács, I. Kézsmárki, N. Kida, H. Murakawa, L. Demkó, Y. Onose, R. Shimano, S. Miyahara, N. Furukawa, and Y. Tokura: arXiv:1109.1597.

11) M. Fiebig: J. Phys. D: Appl. Phys. 38 (2005) R123.

12) W. Eerenstein, N. D. Mathur, and J. F. Scott: Nature (London) 442 (2006) 759.

13) Y. Tanabe, T. Moriya, and S. Sugano: Phys. Rev. Lett. 15 (1965) 1023.

14) T. Moriya: J. Appl. Phys. 39 (1968) 1042.

15) S. Tewari, C. Zhang, J. Toner, and S. Sarma: Phys. Rev. B 78 (2008) 144427.

16) A. Cano: Phys. Rev. B 80 (2009) 180416.

17) M. Stenberg and R. de Sousa: Phys. Rev. B 80 (2009) 094419.

18) M. Mochizuki, N. Furukawa, and N. Nagaosa: Phys. Rev. Lett. 104 (2010) 177206.

19) S. Miyahara and N. Furukawa: J. Phys. Soc. Jpn. 80 (2011) 073708.

20) H. Katsura, N. Nagaosa, and A. V. Balatsky: Phys. Rev. Lett. 95 (2005) 057205.

21) T. Arima: J. Phys. Soc. Jpn. 80 (2011) 052001.

22) T. A. Kaplan and S. D. Mahanti: arXiv:0808.0336v5.

$23)$ L. D. Barron: Molecular Light Scattering and Optical Activity (Cambridge University Press, Cambridge, 2004) 2nd ed.

24) L. D. Landau, E. M. Lifshitz, and L. P. Pitaevskiî: Electrodynamics of Continuous Media (Pergamon Press Ltd., Oxford, 1984) 2nd ed.

25) T. Arima: J. Phys. Soc. Jpn. 76 (2007) 073702.

26) T. Arima, J.-H. Jung, M. Matsubara, M. Kubota, J.-P. He, Y. Kaneko, and Y. Tokura: J. Phys. Soc. Jpn. 74 (2005) 1419.

$27)$ B. R. Cooper, R. J. Elliott, S. J. Nettel, and H. Suhl: Phys. Rev. 127 (1962) 57.

28) In ref. 16, NDD for the non conical cycloidal spin structure due to $\chi_{y x}^{\mathrm{me}}\left(\omega_{ \pm q_{0}}\right)$ is discussed. However, in reality, the $\chi_{y x}^{\mathrm{me}}\left(\omega_{ \pm q_{0}}\right)$ term does not induce NDD owing to $\chi_{y x}^{\mathrm{me}}\left(\omega_{ \pm q_{0}}\right)=$ $-\chi_{x y}^{\mathrm{em}}\left(\omega_{ \pm q_{0}}\right)$ as a consequence of a time-reversal symmetry.

29) Y. Takahashi, R. Shimano, Y. Kaneko, H. Murakawa, and Y. Tokura: Nat. Phys. online publication, 4 Dec. 2011 (DOI 10.1038/NPHYS2161). 